\title{
Minimum supports of eigenfunctions with the second largest eigenvalue of the Star graph*
}

\author{
Vladislav Kabanov \\ Krasovskii Institute of Mathematics and Mechanics \\ Yekaterinburg, Russia \\ Sobolev Institute of Mathematics \\ Novosibirsk, Russia \\ vvk@imm .uran.ru \\ Leonid Shalaginov \\ Chelyabinsk State University \\ Chelyabinsk, Russia
}

Krasovskii Institute of Mathematics and Mechanics Yekaterinburg, Russia

Sobolev Institute of Mathematics

Novosibirsk, Russia

44sh@mail.ru

\author{
Elena V. Konstantinova \\ Sobolev Institute of Mathematics \\ Novosibirsk, Russia \\ Novosibirsk State University \\ Novosibirsk, Russia \\ e_konsta@math.nsc.ru \\ Alexandr Valyuzhenich ${ }^{\dagger}$ \\ Sobolev Institute of Mathematics \\ Novosibirsk, Russia \\ Novosibirsk State University \\ Novosibirsk, Russia \\ graphkiper@mail.ru
}

Submitted: Nov 17, 2019; Accepted: Apr 13, 2020; Published: May 1, 2020

(C) The authors. Released under the CC BY-ND license (International 4.0).

\begin{abstract}
The Star graph $S_{n}, n \geqslant 3$, is the Cayley graph on the symmetric group $S y m_{n}$ generated by the set of transpositions $\{(12),(13), \ldots,(1 n)\}$. In this work we study eigenfunctions of $S_{n}$ corresponding to the second largest eigenvalue $n-2$. For $n \geqslant 8$ and $n=3$, we find the minimum cardinality of the support of an eigenfunction of $S_{n}$ corresponding to the second largest eigenvalue and obtain a characterization of eigenfunctions with the minimum cardinality of the support.
\end{abstract}

Mathematics Subject Classifications: 05C50, 05C25, 05E15, 05B30

*The work is supported by Mathematical Center in Akademgorodok under agreement No. 075-152019-1613 with the Ministry of Science and High Education of the Russian Federation. The reported study was partially funded by RFBR according to the research project 17-51-560008. The second and the fourth authors are partially supported by the program of fundamental scientific research of the SB RAS N I.5.1, project No.0314-2019-0016. The second author is partially supported by RFBR according to the research project 18-501-51021.

${ }^{\dagger}$ Corresponding author. 


\section{Introduction}

The Star graph $S_{n}=\operatorname{Cay}\left(\operatorname{Sym}_{n}, S\right), n \geqslant 3$, is the Cayley graph on the symmetric group $S_{\text {Sym }}$ with the generating set $S=\{(1 i) \mid i \in\{2, \ldots, n\}\}$. It is a connected bipartite $(n-1)$-regular graph of order $n$ ! and diameter $\operatorname{diam}\left(S_{n}\right)=\left\lfloor\frac{3(n-1)}{2}\right\rfloor$ [1]. Since this graph is bipartite it does not contain odd cycles but it does contain all even $l$-cycles where $l=6,8, \ldots, n$ ! (with the sole exception when $l=4$ ) [9] which means that $S_{n}$ is hamiltonian.

The spectrum of the Star graph is integral [5, 12]. More precisely, for $n \geqslant 3$ and for each integer $1 \leqslant k \leqslant n-1$, the values $\pm(n-k)$ are eigenvalues of $S_{n}$; if $n \geqslant 4$, then 0 is an eigenvalue of $S_{n}$. Let $\operatorname{mul}(\lambda)$ denote the multiplicity of an eigenvalue $\lambda$. Since the Star graph is bipartite, $\operatorname{mul}(\mathrm{n}-\mathrm{k})=\operatorname{mul}(-\mathrm{n}+\mathrm{k})$ for each integer $1 \leqslant k \leqslant n$. Moreover, $\pm(n-1)$ are simple eigenvalues of $S_{n}$. A lower bound on multiplicities of the eigenvalues was found as $\left(\begin{array}{c}n-2 \\ k-1\end{array}\right)$ [12], and it was improved as $\left(\begin{array}{c}n-2 \\ n-k-1\end{array}\right)\left(\begin{array}{c}n-1 \\ n-k\end{array}\right)$ [5] for any $1 \leqslant k \leqslant n-1$. Later a method for getting explicit formulas for multiplicities of eigenvalues $\pm(n-k)$ in the Star graphs $S_{n}$ was suggested [2, 10], and the behavior of the eigenvalues multiplicity function of the Star graph $S_{n}$ for eigenvalues $\pm(n-k)$ where $1 \leqslant k \leqslant \frac{n+1}{2}$ was investigated [11]. It was shown that the function has a polynomial behavior on $n$. Computational results showed that the same polynomial behavior of the eigenvalues multiplicity function occurs for any integers $n \geqslant 2$ and $1 \leqslant k \leqslant n$. Moreover, explicit formulas for calculating multiplicities of eigenvalues $\pm(n-k)$ where $2 \leqslant k \leqslant 12$ were found. In particular, $\operatorname{mul}(n-2)=\operatorname{mul}(2-n)=(n-1)(n-2)$.

We investigate the following problem.

Problem 1. For a graph $\Gamma$ and its eigenvalue $\lambda$ to find the minimum cardinality of the support of a $\lambda$-eigenfunction of $\Gamma$.

In many cases Problem 1 is directly related to the problem of finding the minimum possible difference of two combinatorial objects and the problem of finding the minimum cardinality of the bitrades. In more details, these connections are described in $[13,14]$. Problem 1 was studied for the bilinear forms graphs in [17], the cubical distance-regular graphs in [16], the Doob graphs in [3], the Grassmann graphs in [14], the Hamming graphs in $[13,15,18,19,20,21]$, the Johnson graphs in [22] and the Paley graphs in [7]. We note that Problem 1 is completely solved for all eigenvalues only for the Hamming graph and for the Johnson graph. Moreover, even for distance-regular graphs Problem 1 for the second largest eigenvalue is solved only for the Hamming graph, the Johnson graph, the Doob graph and the cubical distance-regular graphs.

In this work we start to investigate Problem 1 for graphs which are not distanceregular and consider Problem 1 for the Star graph $S_{n}$ and its eigenvalue $n-2$. We find the minimum cardinality of the support of $(n-2)$-eigenfunctions of $S_{n}$ and give a characterization of $(n-2)$-eigenfunctions with the minimum cardinality of the support for $n \geqslant 8$ and $n=3$. We also show that for $n \geqslant 8$ and $n=3$ an arbitrary $(n-2)$ eigenfunction of $S_{n}$ with the minimum cardinality of the support is the difference of the characteristic functions of two completely regular codes of covering radius 2 . 
The paper is organized as follows. In Section 2, we introduce basic definitions and give some preliminary results. In Section 3, we reduce Problem 1 for the Star graph $S_{n}$ and its eigenvalue $n-2$ to some extremal problem on the set of real $n \times n$ matrices. In Section 4 , we solve this extremal problem. In Section 5 , for $n \geqslant 8$ and $n=3$ we prove that the minimum cardinality of the support of an $(n-2)$-eigenfunction of $S_{n}$ is $2(n-1)$ ! and give a characterization of eigenfunctions with the minimum cardinality of the support. In Section 6 , for $n \geqslant 8$ and $n=3$ we show that an arbitrary $(n-2)$-eigenfunction of $S_{n}$ with the minimum cardinality of the support is the difference of the characteristic functions of two completely regular codes of covering radius 2 .

\section{Preliminaries}

\subsection{Star graph}

Let $G$ be a group and $S$ be an inverse-closed identity-free generating set in $G$. The Cayley graph on $G$ with the generating set $S$ (denoted by $\operatorname{Cay}(G, S)$ ) is the graph whose vertices are the elements of $G$, and any two elements $x, y \in G$ are adjacent in $\operatorname{Cay}(G, S)$ whenever $x y^{-1} \in S$. For a positive integer $n \geqslant 3$, the Star graph (denoted by $S_{n}$ ) is the Cayley graph on the symmetric group $S_{y} m_{n}$ with the generating set $S=\{(1 i) \mid i \in\{2, \ldots, n\}\}$.

\subsection{Eigenfunctions of graphs}

Let $\Gamma=(V, E)$ be a graph with the adjacency matrix $A(\Gamma)$. The set of neighbors of a vertex $x$ is denoted by $N(x)$. Let $\lambda$ be an eigenvalue of the matrix $A(\Gamma)$. A function $f: V \longrightarrow \mathbb{R}$ is called a $\lambda$-eigenfunction of $\Gamma$ if $f \not \equiv 0$ and the equality

$$
\lambda \cdot f(x)=\sum_{y \in N(x)} f(y)
$$

holds for any $x \in V$. Note that the vector of values of a $\lambda$-eigenfunction is an eigenvector of $A(\Gamma)$ with eigenvalue $\lambda$. The support of a function $f: V \longrightarrow \mathbb{R}$ is the set $\operatorname{Supp}(f)=$ $\{x \in V \mid f(x) \neq 0\}$. For a function $f: V \longrightarrow \mathbb{R}$ denote $E(f)=\{y \in \mathbb{R} \mid y=f(x), x \in V\}$.

Let $u \in\{1, \ldots, n\}$ and $v, w \in\{2, \ldots, n\}$, where $v \neq w$. We define the function $f_{u}^{v, w}: S y m_{n} \longrightarrow \mathbb{R}$ by the following rule:

$$
f_{u}^{v, w}(\pi)= \begin{cases}1, & \text { if } \pi(v)=u \\ -1, & \text { if } \pi(w)=u \\ 0, & \text { otherwise }\end{cases}
$$

The following result is a particular case of Proposition 1 proved in [8].

Lemma 2. Let $u \in\{1, \ldots, n\}$ and $v, w \in\{2, \ldots, n\}$, where $v \neq w$. Then for $n \geqslant 3$, the function $f_{u}^{v, w}$ is an $(n-2)$-eigenfunction of $S_{n}$. 
Denote

$$
\mathcal{F}_{2}=\left\{f_{u}^{2, w} \mid u \in\{2, \ldots, n\}, w \in\{3, \ldots, n\}\right\} .
$$

The following lemma was proved in [8].

Lemma 3 ([8], Lemma 15). For $n \geqslant 3$, the set $\mathcal{F}_{2}$ forms a basis of the eigenspace of $S_{n}$ with eigenvalue $n-2$.

Denote

$$
\mathcal{F}=\left\{f_{u}^{v, w} \mid u \in\{1, \ldots, n\}, v, w \in\{2, \ldots, n\}, v \neq w\right\} .
$$

In Section 5 we prove that if $f$ is an $(n-2)$-eigenfunction of $S_{n}$, then $|\operatorname{Supp}(f)| \geqslant 2(n-1)$ !. Moreover, we prove that $|\operatorname{Supp}(f)|=2(n-1)$ ! if and only if $f=c \cdot \tilde{f}$, where $c$ is a constant and $\tilde{f} \in \mathcal{F}$.

\subsection{Matrices}

Let $M=\left(m_{i, j}\right)$ be a real $n \times n$ matrix. We say that $M$ is special if $M$ is non-zero and the following conditions hold:

1. $m_{i, 1}=0$ for any $i \in\{1, \ldots, n\}$.

2. $m_{1, j}=0$ for any $j \in\{1, \ldots, n\}$.

3. $\sum_{j=1}^{n} m_{i, j}=0$ for any $i \in\{1, \ldots, n\}$.

Example 4. The matrix

$$
M=\left(\begin{array}{cccc}
0 & 0 & 0 & 0 \\
0 & 1 & 0 & -1 \\
0 & 3 & -1 & -2 \\
0 & -1 & -1 & 2
\end{array}\right)
$$

is special.

Let $M=\left(m_{i, j}\right)$ be a real $n \times n$ matrix and let $X$ be a subset of $S y m_{n}$. Denote

$$
g_{M}(n)=\mid\left\{\pi \in \text { Sym }_{n} \mid \sum_{i=1}^{n} m_{i, \pi(i)} \neq 0\right\} \mid
$$

and

$$
g_{M}(X)=\left|\left\{\pi \in X \mid \sum_{i=1}^{n} m_{i, \pi(i)} \neq 0\right\}\right| .
$$

For an $n \times n$ matrix $M$ and $\alpha \in\{1, \ldots, n\}$ denote by $R_{\alpha}$ the $\alpha$-th row of $M$. 


\subsection{Equitable partitions and completely regular codes}

Let $\Gamma=(V, E)$ be a graph. An ordered partition $\left(C_{1}, \ldots, C_{r}\right)$ of $V$ is called equitable if for any $i, j \in\{1, \ldots, r\}$ there is $S_{i, j}$ such that any vertex of $C_{i}$ has exactly $S_{i, j}$ neighbors in $C_{j}$. The matrix $S=\left(S_{i, j}\right)_{i, j \in\{1, \ldots, r\}}$ is called the quotient matrix of the equitable partition. A set $C \subseteq V$ is called a completely regular code in $\Gamma$ if the partition $\left(C^{(0)}, \ldots, C^{(\rho)}\right)$ is equitable, where $C^{(d)}$ is the set of vertices at distance $d$ from $C$ and $\rho$ (the covering radius of $C$ ) is the maximum $d$ for which $C^{(d)}$ is nonempty. In other words, a subset of $V$ is a completely regular code in $\Gamma$ if the distance partition with respect to the subset is equitable. For more information on equitable partitions and completely regular codes see $[4,6]$.

\section{Correspondence between the values of an $(n-2)$-eigenfunction of $S_{n}$ and the diagonals of an $n \times n$ matrix}

In this section, for an arbitrary $(n-2)$-eigenfunction $f$ of $S_{n}$, we introduce a special $n \times n$ matrix $M(f)$ and match the permutations from $S y m_{n}$ with diagonals of $M(f)$ in such a way that the value of $f$ on a permutation $\pi$ is the sum of elements of the corresponding diagonal of $M(f)$.

Let $f$ be an $(n-2)$-eigenfunction of $S_{n}$. By Lemma 3, there exist the numbers $\mu_{i}^{j}(f) \in \mathbb{R}$, where $i \in\{2, \ldots, n\}$ and $j \in\{3, \ldots, n\}$, such that

$$
f=\sum_{\substack{j \in\{3, \ldots, n\} \\ i \in\{2, \ldots, n\}}} \mu_{i}^{j}(f) \cdot f_{i}^{2, j} .
$$

We define the matrix $M(f)=\left(m_{i, j}(f)\right)_{i, j \in\{1, \ldots, n\}}$ by the following rule:

$$
m_{i, j}(f)= \begin{cases}-\mu_{i}^{j}(f), & \text { if } i>1 \text { and } j>2 \\ \sum_{s=3}^{n} \mu_{i}^{s}(f), & \text { if } i>1 \text { and } j=2 \\ 0, & \text { if } i=1 \text { or } j=1\end{cases}
$$

Lemma 5. Let $f$ be an $(n-2)$-eigenfunction of $S_{n}$. Then

$$
f(\pi)=\sum_{i=1}^{n} m_{i, \pi^{-1}(i)}(f)
$$

for any $\pi \in$ Sym $_{n}$.

Proof. For $i \in\{2, \ldots, n\}$ denote

$$
f_{i}=\sum_{j=3}^{n} \mu_{i}^{j}(f) \cdot f_{i}^{2, j}
$$


By the definition of $f_{u}^{v, w}$ we have

$$
f_{i}(\pi)= \begin{cases}-\mu_{i}^{j}(f), & \text { if } \pi(j)=i \text { and } j \in\{3, \ldots, n\} \\ \sum_{s=3}^{n} \mu_{i}^{s}(f), & \text { if } \pi(2)=i \\ 0, & \text { if } \pi(1)=i\end{cases}
$$

Using the equalities (1) and (2), we see that $f_{i}(\pi)=m_{i, \pi^{-1}(i)}(f)$ for any $i \in\{2, \ldots, n\}$ and $\pi \in$ Sym $_{n}$. Then

$$
f(\pi)=\sum_{i=2}^{n} f_{i}(\pi)=\sum_{i=2}^{n} m_{i, \pi^{-1}(i)}(f)
$$

for any $\pi \in S y m_{n}$. Since $m_{1, \pi^{-1}(1)}(f)=0$ due to the definition of $M(f)$, we obtain that

$$
f(\pi)=\sum_{i=1}^{n} m_{i, \pi^{-1}(i)}(f) .
$$

Lemma 6. Let $f$ be an $(n-2)$-eigenfunction of $S_{n}$. Then

$$
E(f)=\left\{\sum_{i=1}^{n} m_{i, \pi(i)}(f) \mid \pi \in S y m_{n}\right\} .
$$

Proof. We note that

$$
\left\{\sum_{i=1}^{n} m_{i, \pi(i)}(f) \mid \pi \in \text { Sym }_{n}\right\}=\left\{\sum_{i=1}^{n} m_{i, \pi^{-1}(i)}(f) \mid \pi \in \text { Sym }_{n}\right\} .
$$

Then by Lemma 5 we have

$$
E(f)=\left\{\sum_{i=1}^{n} m_{i, \pi(i)}(f) \mid \pi \in \operatorname{Sym}_{n}\right\} .
$$

Using Lemma 6 and the definition of $g_{M}(n)$, we immediately obtain the following result.

Lemma 7. Let $f$ be an $(n-2)$-eigenfunction of $S_{n}$. Then $|\operatorname{Supp}(f)|=g_{M(f)}(n)$.

\section{Extremal problem on the set of all special $n \times n$ matrices}

In view of Lemma 7, in order to solve Problem 1 for the Star graph $S_{n}$ and its eigenvalue $n-2$, it suffices to find the minimum value of $g_{M(f)}(n)$, where $n$ is fixed and $f$ is an arbitary $(n-2)$-eigenfunction of $S_{n}$. Since the matrix $M(f)$ is special, in this section we focus on the following extremal problem formulated for the class of special matrices.

Problem 8. Given a positive integer $n$, to find the minimum value of $g_{M}(n)$ for the class of special $n \times n$ matrices $M$. 
In this section, we solve Problem 8 (see Theorem 1 ) and prove that $g_{M}(n) \geqslant 2(n-1)$ ! holds for any special $n \times n$ matrix $M$ with $n \geqslant 8$ or $n=3$. We then prove that this bound is tight and classify the special matrices in the equality case. This finally leads to a solution of Problem 1 for the Star graph $S_{n}$ and its eigenvalue $n-2$ (see Theorem 20).

Let $M=\left(m_{i, j}\right)$ be a real $n \times n$ matrix and let $\left(A_{1}, \ldots, A_{t}\right)$ be a partition of $\{1, \ldots, n\}$, where $t \geqslant 2$. Let $\alpha, \beta \in\{1, \ldots, n\}$ and $\alpha \neq \beta$. We say that $R_{\alpha}$ and $R_{\beta}$ have the $\left(A_{1}, \ldots, A_{t}\right)$-property if for any $k, m \in\{1, \ldots, t\}, k \neq m$, and for any $a \in A_{k}, b \in A_{m}$ the condition

$$
m_{\alpha, a}+m_{\beta, b} \neq m_{\alpha, b}+m_{\beta, a}
$$

holds.

Example 9. Let us consider the matrix

$$
M=\left(\begin{array}{cccc}
0 & 0 & 0 & 0 \\
0 & 1 & -1 & 0 \\
2 & 2 & 0 & 0 \\
0 & 0 & 3 & 3
\end{array}\right) .
$$

1. The rows $R_{1}$ and $R_{2}$ have $\left(A_{1}, A_{2}, A_{3}\right)$-property, where $A_{1}=\{1,4\}, A_{2}=\{2\}$ and $A_{3}=\{3\}$.

2. The rows $R_{1}$ and $R_{3}$ have $\left(A_{1}, A_{2}\right)$-property, where $A_{1}=\{1,2\}$ and $A_{2}=\{3,4\}$.

3. The rows $R_{3}$ and $R_{4}$ have $\left(A_{1}, A_{2}\right)$-property, where $A_{1}=\{1,2\}$ and $A_{2}=\{3,4\}$.

In Lemmas 10 and 11, we obtain lower bounds for $g_{M}(n)$, where $M$ is an arbitrary real matrix having a pair of rows with the $\left(A_{1}, \ldots, A_{t}\right)$-property.

Let $\left\{a_{1}, \ldots, a_{\ell}\right\}$ and $\left\{\alpha_{1}, \ldots, \alpha_{\ell}\right\}$ be subsets of $\{1, \ldots, n\}$ for some $1 \leqslant \ell \leqslant n$. Denote by $S_{a_{1}, \ldots, a_{\ell}}^{\alpha_{1}, \ldots, \alpha_{\ell}}$ the set of permutations $\pi \in S y m_{n}$ such that $\pi\left(\alpha_{i}\right)=a_{i}$ for any $i \in\{1, \ldots, \ell\}$.

Lemma 10. Let $M=\left(m_{i, j}\right)$ be a real $n \times n$ matrix. Let $\left\{c_{1}, \ldots, c_{h}, a, b\right\},\left\{\gamma_{1}, \ldots, \gamma_{h}, \alpha, \beta\right\}$ be subsets of $\{1, \ldots, n\}$ for some $0 \leqslant h \leqslant n-2$. Suppose $R_{\alpha}$ and $R_{\beta}$ have the $\left(A_{1}, \ldots, A_{t}\right)$ property and $a \in A_{k}, b \in A_{m}$ for some $k, m \in\{1, \ldots, t\}$ and $k \neq m$. Then

$$
g_{M}\left(S_{c_{1}, \ldots, c_{h}, a, b}^{\gamma_{1}, \ldots, \gamma_{h}, \alpha, \beta} \cup S_{c_{1}, \ldots, c_{h}, b, a}^{\gamma_{1}, \ldots, \gamma_{h}, \alpha, \beta}\right) \geqslant(n-h-2) ! .
$$

Proof. Denote $Y_{1}=S_{c_{1}, \ldots, c_{h}, a, b}^{\gamma_{1}, \ldots, \gamma_{h}, \alpha, \beta}$ and $Y_{2}=S_{c_{1}, \ldots, c_{h}, b, a}^{\gamma_{1}, \ldots, \gamma_{h}, \alpha, \beta}$. We note that for any permutation $\pi \in Y_{1}$ there is a unique permutation $\pi^{\prime} \in Y_{2}$ such that $\pi(s)=\pi^{\prime}(s)$ for any $s \in$ $\{1,2, \ldots, n\} \backslash\{\alpha, \beta\}$. Then

$$
\sum_{i=1}^{n} m_{i, \pi(i)}-\sum_{i=1}^{n} m_{i, \pi^{\prime}(i)}=m_{\alpha, a}+m_{\beta, b}-m_{\alpha, b}-m_{\beta, a}
$$

for any $\pi \in Y_{1}$. Since $R_{\alpha}$ and $R_{\beta}$ have the $\left(A_{1}, \ldots, A_{t}\right)$-property, $a \in A_{k}$ and $b \in A_{m}$, we have

$$
\sum_{i=1}^{n} m_{i, \pi(i)} \neq \sum_{i=1}^{n} m_{i, \pi^{\prime}(i)}
$$


Therefore, $\sum_{i=1}^{n} m_{i, \pi(i)} \neq 0$ or $\sum_{i=1}^{n} m_{i, \pi^{\prime}(i)} \neq 0$ for any $\pi \in Y_{1}$. Using the equality

$$
\left|Y_{1}\right|=\left|Y_{2}\right|=(n-h-2) !
$$

we obtain

$$
g_{M}\left(Y_{1} \cup Y_{2}\right) \geqslant(n-h-2) !
$$

Lemma 11. Let $M$ be a real $n \times n$ matrix, $\alpha, \beta \in\{1, \ldots, n\}$ and $\alpha \neq \beta$. Suppose $R_{\alpha}$ and $R_{\beta}$ have the $\left(A_{1}, \ldots, A_{t}\right)$-property. Then

$$
g_{M}(n) \geqslant\left(\sum_{1 \leqslant k<m \leqslant t}\left|A_{k}\right| \cdot\left|A_{m}\right|\right) \cdot(n-2) !
$$

Proof. Let $k, m \in\{1, \ldots, t\}$ and $k<m$. Let us consider arbitrary $a \in A_{k}$ and $b \in A_{m}$. Lemma 10 implies that $g_{M}\left(S_{a, b}^{\alpha, \beta} \cup S_{b, a}^{\alpha, \beta}\right) \geqslant(n-2)$ !. Denote

$$
X=\bigcup_{\substack{1 \leqslant k<m \leqslant t \\ a \in A_{k}, b \in A_{m}}}\left(S_{a, b}^{\alpha, \beta} \cup S_{b, a}^{\alpha, \beta}\right)
$$

Then we have

$$
g_{M}(X)=\sum_{\substack{1 \leqslant k<m \leqslant t \\ a \in A_{k}, b \in A_{m}}} g_{M}\left(S_{a, b}^{\alpha, \beta} \cup S_{b, a}^{\alpha, \beta}\right) \geqslant\left(\sum_{1 \leqslant k<m \leqslant t}\left|A_{k}\right| \cdot\left|A_{m}\right|\right) \cdot(n-2) ! .
$$

Thus, we obtain

$$
g_{M}(n) \geqslant g_{M}(X) \geqslant\left(\sum_{1 \leqslant k<m \leqslant t}\left|A_{k}\right| \cdot\left|A_{m}\right|\right) \cdot(n-2) !
$$

Lemma 12. Let $n=n_{1}+\ldots+n_{t}$, where $n_{i} \in \mathbb{N}$ for any $i \in\{1, \ldots, t\}, n_{1} \geqslant \ldots \geqslant n_{t}$, $n \geqslant 7$ and $t \geqslant 3$. Then either

$$
\sum_{1 \leqslant k<m \leqslant t} n_{k} n_{m}>2(n-1)
$$

or

$$
t=3, n_{1}=n-2 \text { and } n_{2}=n_{3}=1 .
$$

Proof. Denote

$$
S=\sum_{1 \leqslant k<m \leqslant t} n_{k} n_{m}
$$

We consider three cases.

In the first case we suppose that $3 \leqslant n_{1} \leqslant n-3$. Then we have

$$
S \geqslant n_{1}\left(n_{2}+\ldots+n_{t}\right)+n_{2} n_{3} \geqslant 3(n-3)+1>2(n-1) .
$$


In the second case we suppose that $n_{1} \geqslant n-2$. Since $t \geqslant 3$, it is possible only if $t=3$, $n_{1}=n-2$ and $n_{2}=n_{3}=1$.

In the third case we suppose that $n_{1} \leqslant 2$. Let $i=\left|\left\{1 \leqslant k \leqslant t \mid n_{k}=2\right\}\right|$.

If $i \geqslant 2$, then $n_{1}=n_{2}=2$ and

$$
S \geqslant n_{1}\left(n_{2}+\ldots+n_{t}\right)+n_{2}\left(n_{3}+\ldots+n_{t}\right)=4 n-12>2(n-1) .
$$

If $n-i \geqslant 3$, then $n_{t}=n_{t-1}=n_{t-2}=1$ and

$S \geqslant n_{t}\left(n_{1}+\ldots+n_{t-1}\right)+n_{t-1}\left(n_{1}+\ldots+n_{t-2}\right)+n_{t-2}\left(n_{1}+\ldots+n_{t-3}\right)=3 n-6>2(n-1)$.

Since $n \geqslant 7, i \geqslant 2$ or $n-i \geqslant 3$ and we obtain the case considered above.

Let $M=\left(m_{i, j}\right)$ be a real $n \times n$ matrix and $\alpha \in\{1, \ldots, n\}$. Let $x, y \in \mathbb{R}$ and $r_{1}, r_{2}, s \in\{2, \ldots, n\}$, where $x, y \neq 0$ and $r_{1} \neq r_{2}$. We say that $R_{\alpha}$ is the $\left(x, r_{1}, r_{2}\right)$-row if $m_{\alpha, r_{1}}=x, m_{\alpha, r_{2}}=-x$ and $m_{\alpha, j}=0$ for any $j \in\{1, \ldots, n\} \backslash\left\{r_{1}, r_{2}\right\}$. We say that $R_{\alpha}$ is the $(y, s)$-row if $m_{\alpha, s}=(n-2) y, m_{\alpha, 1}=0$ and $m_{\alpha, j}=-y$ for any $j \in\{1, \ldots, n\} \backslash\{1, s\}$.

Example 13. Let

$$
M=\left(\begin{array}{cccc}
0 & 0 & 0 & 0 \\
0 & 1 & 0 & -1 \\
0 & 0 & 0 & 0 \\
0 & -2 & 4 & -2
\end{array}\right) .
$$

Then $R_{2}$ is the $(1,2,4)$-row and $R_{4}$ is the $(2,3)$-row.

Lemma 14. Let $M$ be a special $n \times n$ matrix, $g_{M}(n) \leqslant 2(n-1) !, n \geqslant 7$ and $\alpha \in\{1, \ldots, n\}$. Suppose $R_{\alpha}$ is a non-zero row. Then $R_{\alpha}$ is the $\left(x, r_{1}, r_{2}\right)$-row or the $(y, s)$-row.

Proof. Suppose that $R_{\alpha}$ consists of the distinct elements $z_{1}, \ldots, z_{t}$ each of them $z_{k}$, where $1 \leqslant k \leqslant t$, occurs $n_{k}$ times in $R_{\alpha}$. Without loss of generality, we assume that $n_{1} \geqslant \ldots \geqslant$ $n_{t}$. If $t \leqslant 2$, then by the definition of special matrix we obtain that all elements of $R_{\alpha}$ are zeros. So, we can assume that $t \geqslant 3$.

For $k \in\{1, \ldots, t\}$ denote

$$
A_{k}=\left\{j \in\{1, \ldots, n\} \mid m_{\alpha, j}=z_{k}\right\} .
$$

We note that $\left|A_{k}\right|=n_{k}$ for any $k \in\{1, \ldots, t\}$. Since $M$ is special, all elements of $R_{1}$ are zeros. Hence $R_{1}$ and $R_{\alpha}$ have $\left(A_{1}, \ldots, A_{t}\right)$-property. Lemma 11 implies that

$$
g_{M}(n) \geqslant\left(\sum_{1 \leqslant k<m \leqslant t} n_{k} n_{m}\right) \cdot(n-2) !
$$

On the other hand, we have $g_{M}(n) \leqslant 2(n-1)$ !. So

$$
\sum_{1 \leqslant k<m \leqslant t} n_{k} n_{m} \leqslant 2(n-1) \text {. }
$$

Recall that in the beginning of the proof we assumed $t \geqslant 3$. Then by Lemma 12 we obtain that $t=3, n_{1}=n-2$ and $n_{2}=n_{3}=1$. Therefore, by the definition of special matrix we obtain that $R_{\alpha}$ is the $\left(x, r_{1}, r_{2}\right)$-row or the $(y, s)$-row. 
Lemma 15. Let $M$ be a special $n \times n$ matrix and $g_{M}(n) \leqslant 2(n-1)$ !, where $n \geqslant 8$. Suppose $\alpha, \beta \in\{1, \ldots, n\}, \alpha \neq \beta$ and $R_{\alpha} \neq R_{\beta}$. Then there exists a partition $(A, B)$ of $\{1, \ldots, n\}$ such that $R_{\alpha}$ and $R_{\beta}$ have the $(A, B)$-property and $|A|=2$.

Proof. Firstly, we prove that $R_{\alpha}$ and $R_{\beta}$ have the $(A, B)$-property, where $|A| \in\{2,3,4\}$. For a set $X \subseteq\{1, \ldots, n\}$ denote $\bar{X}=\{1, \ldots, n\} \backslash X$. By Lemma 14 we have four cases for $R_{\alpha}$ and $R_{\beta}$.

Case 1. Suppose $R_{\alpha}$ or $R_{\beta}$ is a zero row. Without loss of generality, we assume that $R_{\alpha}$ is a zero row. Let us consider two subcases.

1.1. $R_{\beta}$ is the $\left(x, r_{1}, r_{2}\right)$-row. Let $A=\left\{r_{1}, r_{2}\right\}$ and $B=\bar{A}$. Then $R_{\alpha}$ and $R_{\beta}$ have the $(A, B)$-property and $|A|=2$.

1.2. $R_{\beta}$ is the $(y, s)$-row. Let $A=\{1, s\}$ and $B=\bar{A}$. Then $R_{\alpha}$ and $R_{\beta}$ have the $(A, B)$-property and $|A|=2$.

Case 2. Suppose $R_{\alpha}$ is the $\left(x_{1}, r_{1}, r_{2}\right)$-row and $R_{\beta}$ is the $\left(x_{2}, r_{3}, r_{4}\right)$-row. Let us consider four subcases depending on $\left|\left\{r_{1}, r_{2}\right\} \cap\left\{r_{3}, r_{4}\right\}\right|$.

2.1. $\left\{r_{1}, r_{2}\right\} \cap\left\{r_{3}, r_{4}\right\}=\emptyset$. Let $A=\left\{r_{1}, r_{2}, r_{3}, r_{4}\right\}$ and $B=\bar{A}$. Then $R_{\alpha}$ and $R_{\beta}$ have the $(A, B)$-property and $|A|=4$.

2.2. $\left|\left\{r_{1}, r_{2}\right\} \cap\left\{r_{3}, r_{4}\right\}\right|=1$ and $x_{1} \neq x_{2}$. Without loss of generality, we assume that $r_{1}=r_{3}$. Let $A=\left\{r_{1}, r_{2}, r_{4}\right\}$ and $B=\bar{A}$. Then $R_{\alpha}$ and $R_{\beta}$ have the $(A, B)$-property and $|A|=3$.

2.3. $\left|\left\{r_{1}, r_{2}\right\} \cap\left\{r_{3}, r_{4}\right\}\right|=1$ and $x_{1}=x_{2}$. Without loss of generality, we assume that $r_{1}=r_{3}$. Let $A=\left\{r_{2}, r_{4}\right\}$ and $B=\bar{A}$. Then $R_{\alpha}$ and $R_{\beta}$ have the $(A, B)$-property and $|A|=2$.

2.4. $\left|\left\{r_{1}, r_{2}\right\} \cap\left\{r_{3}, r_{4}\right\}\right|=2$. Without loss of generality, we assume that $r_{1}=r_{3}$ and $r_{2}=r_{4}$. Since $R_{\alpha} \neq R_{\beta}$, we have $x_{1} \neq x_{2}$. Let $A=\left\{r_{1}, r_{2}\right\}$ and $B=\bar{A}$. Then $R_{\alpha}$ and $R_{\beta}$ have the $(A, B)$-property and $|A|=2$.

Case 3. Suppose $R_{\alpha}$ is the $\left(y_{1}, s_{1}\right)$-row and $R_{\beta}$ is the $\left(y_{2}, s_{2}\right)$-row. Let us consider three subcases.

3.1. $s_{1} \neq s_{2}$ and $y_{1} \neq y_{2}$. Let $A=\left\{1, s_{1}, s_{2}\right\}$ and $B=\bar{A}$. Then $R_{\alpha}$ and $R_{\beta}$ have the $(A, B)$-property and $|A|=3$.

3.2. $s_{1} \neq s_{2}$ and $y_{1}=y_{2}$. Let $A=\left\{s_{1}, s_{2}\right\}$ and $B=\bar{A}$. Then $R_{\alpha}$ and $R_{\beta}$ have the $(A, B)$-property and $|A|=2$.

3.3. $s_{1}=s_{2}$. Since $R_{\alpha} \neq R_{\beta}$, we have $y_{1} \neq y_{2}$. Let $A=\left\{1, s_{1}\right\}$ and $B=\bar{A}$. Then $R_{\alpha}$ and $R_{\beta}$ have the $(A, B)$-property and $|A|=2$.

Case 4. Suppose $R_{\alpha}$ is the $\left(x, r_{1}, r_{2}\right)$-row and $R_{\beta}$ is the $(y, s)$-row (the case when $R_{\alpha}$ is the $(y, s)$-row and $R_{\beta}$ is the $\left(x, r_{1}, r_{2}\right)$-row is similar). Let us consider three subcases.

4.1. $s \notin\left\{r_{1}, r_{2}\right\}$. Let $A=\left\{1, r_{1}, r_{2}, s\right\}$ and $B=\bar{A}$. Then $R_{\alpha}$ and $R_{\beta}$ have the $(A, B)$-property and $|A|=4$.

4.2. $s \in\left\{r_{1}, r_{2}\right\}$ and $x \neq(n-1) y$. Without loss of generality, we assume that $s=r_{1}$. Let $A=\left\{1, r_{1}, r_{2}\right\}$ and $B=\bar{A}$. Then $R_{\alpha}$ and $R_{\beta}$ have the $(A, B)$-property and $|A|=3$.

4.3. $s \in\left\{r_{1}, r_{2}\right\}$ and $x=(n-1) y$. Without loss of generality, we assume that $s=r_{1}$. Let $A=\left\{1, r_{2}\right\}$ and $B=\bar{A}$. Then $R_{\alpha}$ and $R_{\beta}$ have the $(A, B)$-property and $|A|=2$.

Thus, we prove that $R_{\alpha}$ and $R_{\beta}$ have the $(A, B)$-property, where $|A| \in\{2,3,4\}$. Hence by Lemma 11 we obtain that $g_{M}(n) \geqslant|A| \cdot|B| \cdot(n-2)$ !. On the other hand, we have 
$g_{M}(n) \leqslant 2(n-1)$ !. Therefore $|A| \cdot|B| \leqslant 2(n-1)$. So, $|A|=2$ and the cases when $|A| \in\{3,4\}$ do not hold. The lemma is proved.

Let $M$ be an $n \times n$ matrix and $\theta \in\{1, \ldots, n\}$. We say that $M$ is $\theta$-uniform if $R_{\alpha}=R_{\beta}$ for any $\alpha, \beta \in\{1, \ldots, n\} \backslash \theta$.

Lemma 16. Let $M$ be a special $n \times n$ matrix and $g_{M}(n) \leqslant 2(n-1)$ !, where $n \geqslant 8$ or $n=3$. Then $M$ is $\theta$-uniform for some $\theta \in\{1, \ldots, n\}$.

Proof. Firstly, let us consider the case $n=3$. Since $M$ is special, any non-zero row of $M$ is the $(x, 2,3)$-row. Hence either $M$ is $\theta$-uniform for some $\theta \in\{1,2,3\}$ or $R_{2}$ and $R_{3}$ are $\left(x_{1}, 2,3\right)$-row and $\left(x_{2}, 2,3\right)$-row, where $x_{1} \neq x_{2}$. In the last case we have $g_{M}(n)=6$ and we obtain a contradiction with $g_{M}(n) \leqslant 2(n-1)$ !. In what follows, in this lemma we assume that $n \geqslant 8$.

Suppose that $M$ is not $\theta$-uniform for any $\theta \in\{1, \ldots, n\}$. Then there exist four rows $R_{\alpha}, R_{\beta}, R_{\gamma}$ and $R_{\delta}$ such that $R_{\alpha} \neq R_{\beta}$ and $R_{\gamma} \neq R_{\delta}$.

Since $R_{\alpha} \neq R_{\beta}$, by Lemma 15 we obtain that $R_{\alpha}$ and $R_{\beta}$ have $(A, B)$-property, where $|A|=2$. Let $a \in A$ and $b \in B$. Lemma 10 implies that $g_{M}\left(S_{a, b}^{\alpha, \beta} \cup S_{b, a}^{\alpha, \beta}\right) \geqslant(n-2)$ !. Denote

$$
X_{1}=\bigcup_{a \in A, b \in B}\left(S_{a, b}^{\alpha, \beta} \cup S_{b, a}^{\alpha, \beta}\right)
$$

Then we have

$$
g_{M}\left(X_{1}\right)=\sum_{a \in A, b \in B} g_{M}\left(S_{a, b}^{\alpha, \beta} \cup S_{b, a}^{\alpha, \beta}\right) \geqslant|A| \cdot|B| \cdot(n-2) !=2(n-2)(n-2) !
$$

Since $R_{\gamma} \neq R_{\delta}$, by Lemma 15 we obtain that $R_{\gamma}$ and $R_{\delta}$ have $(C, D)$-property, where $|C|=2$. Let $c \in C$ and $d \in D$. Let us consider arbitrary $b^{\prime}, b^{\prime \prime} \in B \backslash\{c, d\}$, where $b^{\prime} \neq b^{\prime \prime}$. Lemma 10 implies that

$$
g_{M}\left(S_{b^{\prime}, b^{\prime \prime}, c, d}^{\alpha, \beta, \gamma} \cup S_{b^{\prime}, b^{\prime \prime}, d, c}^{\alpha, \beta, \gamma, \delta}\right) \geqslant(n-4) !
$$

Denote

$$
X_{2}=\bigcup_{\substack{c \in C, d \in D \\ b^{\prime}, b^{\prime \prime} \in B \backslash\{c, d\}, b^{\prime} \neq b^{\prime \prime}}}\left(S_{b^{\prime}, b^{\prime \prime}, c, d}^{\alpha, \beta, \gamma} \cup S_{b^{\prime}, b^{\prime \prime}, d, c}^{\alpha, \gamma, \gamma}\right) .
$$

Then we have

$$
\begin{aligned}
& g_{M}\left(X_{2}\right)=\sum_{\substack{c \in C, d \in D \\
b^{\prime}, b^{\prime \prime} \in B \backslash\{c, d\}, b^{\prime} \neq b^{\prime \prime}}} g_{M}\left(S_{b^{\prime}, b^{\prime \prime}, c, d}^{\alpha, \beta, \gamma} \cup S_{b^{\prime}, b^{\prime \prime}, d, c}^{\alpha, \beta, \delta}\right) \geqslant \\
& \geqslant|C| \cdot|D| \cdot(|B|-2) \cdot(|B|-3) \cdot(n-4) != \\
& =2(n-2)(n-4)(n-5)(n-4) ! .
\end{aligned}
$$

Note that $X_{1} \cap X_{2}=\emptyset$. Therefore, $g_{M}\left(X_{1} \cup X_{2}\right)=g_{M}\left(X_{1}\right)+g_{M}\left(X_{2}\right)$. Using (3) and (4), we obtain that $g_{M}\left(X_{1} \cup X_{2}\right)>2(n-1)$ !. Thus, $g_{M}(n) \geqslant g_{M}\left(X_{1} \cup X_{2}\right)>2(n-1)$ ! and we obtain a contradiction with $g_{M}(n) \leqslant 2(n-1)$ !. 
Let $x \in \mathbb{R}, x \neq 0$ and $p_{1}, p_{2} \in\{2, \ldots, n\}, p_{1} \neq p_{2}$. We say that an $n \times n$ matrix $M=\left(m_{i, j}\right)$ is the $\left(x, p_{1}, p_{2}\right)$-matrix if

$$
m_{i, j}= \begin{cases}x, & \text { if } j=p_{1} \text { and } i>1 \\ -x, & \text { if } j=p_{2} \text { and } i>1 \\ 0, & \text { otherwise }\end{cases}
$$

Example 17. The matrix

$$
M=\left(\begin{array}{cccc}
0 & 0 & 0 & 0 \\
0 & 3 & 0 & -3 \\
0 & 3 & 0 & -3 \\
0 & 3 & 0 & -3
\end{array}\right)
$$

is the $(3,2,4)$-matrix.

We say that an $n \times n$ matrix $M$ belongs to the set $\mathcal{M}_{1}(n)$ if $M$ is the $\left(x, p_{1}, p_{2}\right)$-matrix for some $x \in \mathbb{R}, x \neq 0$ and $p_{1}, p_{2} \in\{2, \ldots, n\}, p_{1} \neq p_{2}$.

Let $x \in \mathbb{R}, x \neq 0$ and $q_{1}, q_{2}, \tau \in\{2, \ldots, n\}, q_{1} \neq q_{2}$. We say that an $n \times n$ matrix $M$ is the $\left(x, q_{1}, q_{2}, \tau\right)$-matrix if

$$
m_{i, j}= \begin{cases}x, & \text { if } i=\tau \text { and } j=q_{1} \\ -x, & \text { if } i=\tau \text { and } j=q_{2} \\ 0, & \text { otherwise. }\end{cases}
$$

Example 18. The matrix

$$
M=\left(\begin{array}{cccc}
0 & 0 & 0 & 0 \\
0 & 0 & 0 & 0 \\
0 & 5 & 0 & -5 \\
0 & 0 & 0 & 0
\end{array}\right)
$$

is the $(5,2,4,3)$-matrix.

We say that an $n \times n$ matrix $M$ belongs to the set $\mathcal{M}_{2}(n)$ if $M$ is the $\left(x, q_{1}, q_{2}, \tau\right)$-matrix for some $x \in \mathbb{R}, x \neq 0$ and $q_{1}, q_{2}, \tau \in\{2, \ldots, n\}, q_{1} \neq q_{2}$.

Now we prove the main theorem of this section.

Theorem 19. Let $M$ be a special $n \times n$ matrix, where $n \geqslant 8$ or $n=3$. Then $g_{M}(n) \geqslant$ $2(n-1)$ !. Moreover, $g_{M}(n)=2(n-1)$ ! if and only if $M \in \mathcal{M}_{1}(n)$ or $M \in \mathcal{M}_{2}(n)$.

Proof. One can verify that if $M \in \mathcal{M}_{1}(n)$ or $M \in \mathcal{M}_{2}(n)$, then $g_{M}(n)=2(n-1)$ !.

Suppose that $g_{M}(n) \leqslant 2(n-1)$ !. Let us prove that $M \in \mathcal{M}_{1}(n)$ or $M \in \mathcal{M}_{2}(n)$. Lemma 16 implies that $M$ is $\theta$-uniform for some $\theta \in\{1, \ldots, n\}$. We consider two cases.

Case 1. Suppose $\theta=1$. Then $R_{2}=R_{3}=\ldots=R_{n}$. By Lemma $14, R_{2}$ is the $\left(x, r_{1}, r_{2}\right)$-row or the $(y, s)$-row. If $R_{2}$ is the $\left(x, r_{1}, r_{2}\right)$-row, then $M$ is the $\left(x, r_{1}, r_{2}\right)$-matrix. So, in this subcase $M \in \mathcal{M}_{1}(n)$. If $R_{2}$ is the $(y, s)$-row, then $g_{M}(n)=(n-1) \cdot(n-1)$ ! and we obtain a contradiction with $g_{M}(n) \leqslant 2(n-1)$ !. 
Case 2. Suppose $\theta>1$. By Lemma $14, R_{\theta}$ is the $\left(x, r_{1}, r_{2}\right)$-row or the $(y, s)$-row. If $R_{\theta}$ is the $\left(x, r_{1}, r_{2}\right)$-row, then $M$ is the $\left(x, r_{1}, r_{2}, \theta\right)$-matrix. So, in this subcase $M \in \mathcal{M}_{2}(n)$. If $R_{\theta}$ is the $(y, s)$-row, then $g_{M}(n)=(n-1) \cdot(n-1)$ ! and we obtain a contradiction with $g_{M}(n) \leqslant 2(n-1) !$.

\section{Main Theorem}

In this section we prove the main theorem of this paper.

Theorem 20. Let $f$ be an $(n-2)$-eigenfunction of $S_{n}$, where $n \geqslant 8$ or $n=3$. Then $|\operatorname{Supp}(f)| \geqslant 2(n-1)$ !. Moreover, $|\operatorname{Supp}(f)|=2(n-1)$ ! if and only if $f=c \cdot \tilde{f}$, where $c$ is a real non-zero constant and $\tilde{f} \in \mathcal{F}$.

Proof. Lemma 7 implies that $|S u p p(f)|=g_{M(f)}(n)$. We note that $M(f)$ is special. Then by Theorem 19 we obtain that $g_{M(f)}(n) \geqslant 2(n-1)$ !. Therefore $|\operatorname{Supp}(f)| \geqslant 2(n-1)$ !. Moreover, $g_{M(f)}(n)=2(n-1)$ ! if and only if $M(f) \in \mathcal{M}_{1}(n)$ or $M(f) \in \mathcal{M}_{2}(n)$. We consider two cases.

Suppose that $M(f) \in \mathcal{M}_{1}(n)$. Then $M(f)$ is the $\left(x, p_{1}, p_{2}\right)$-matrix. Using Lemma 5 , we have

$$
f(\pi)= \begin{cases}x, & \text { if } \pi\left(p_{2}\right)=1 \\ -x, & \text { if } \pi\left(p_{1}\right)=1 \\ 0, & \text { otherwise }\end{cases}
$$

So, in this case $f=x \cdot f_{1}^{p_{2}, p_{1}}$.

Suppose that $M(f) \in \mathcal{M}_{2}(n)$. Then $M(f)$ is the $\left(x, q_{1}, q_{2}, \tau\right)$-matrix. Using Lemma 5 , we have

$$
f(\pi)= \begin{cases}x, & \text { if } \pi\left(q_{1}\right)=\tau \\ -x, & \text { if } \pi\left(q_{2}\right)=\tau \\ 0, & \text { otherwise }\end{cases}
$$

So, in this case $f=x \cdot f_{\tau}^{q_{1}, q_{2}}$.

\section{Correspondence between the extremal $(n-2)$-eigenfunctions and completely regular codes}

Lemma 21. Let $\alpha \in\{2, \ldots, n\}, a \in\{1, \ldots, n\}$ and $n \geqslant 3$. Then the set $S_{a}^{\alpha}$ is a completely regular code of covering radius 2 in $S_{n}$.

Proof. Firstly, we note that $\left(S_{a}^{\alpha}\right)^{(1)}=S_{a}^{1}$,

$$
\left(S_{a}^{\alpha}\right)^{(2)}=\bigcup_{\beta \in\{1, \ldots, n\} \backslash\{1, \alpha\}} S_{a}^{\beta}
$$


and $\left(S_{a}^{\alpha}\right)^{(3)}=\emptyset$. On the other hand, the partition $\left(\left(S_{a}^{\alpha}\right)^{(0)},\left(S_{a}^{\alpha}\right)^{(1)},\left(S_{a}^{\alpha}\right)^{(2)}\right)$ has the quotient matrix

$$
\left(\begin{array}{ccc}
n-2 & 1 & 0 \\
1 & 0 & n-2 \\
0 & 1 & n-2
\end{array}\right) .
$$

So, $S_{a}^{\alpha}$ is a completely regular code of covering radius 2 in $S_{n}$.

For a set $A \subseteq S y m_{n}$ we define the characteristic function $\chi_{A}$ of $A$ in $S y m_{n}$ as follows:

$$
\chi_{A}(\pi)= \begin{cases}1, & \text { if } \pi \in A \\ 0, & \text { otherwise }\end{cases}
$$

Using Theorem 20 and the definition of $f_{u}^{v, w}$, we immediately obtain the following result.

Lemma 22. Let $f$ be an $(n-2)$-eigenfunction of $S_{n}$ and $|\operatorname{Supp}(f)|=2(n-1)$ !, where $n \geqslant 8$ or $n=3$. Then

$$
f=c \cdot\left(\chi_{S_{u}^{v}}-\chi_{S_{u}^{w}}\right),
$$

where $c$ is a real non-zero constant, $u \in\{1, \ldots, n\}, v, w \in\{2, \ldots, n\}$ and $v \neq w$.

Thus, Lemma 21 and Lemma 22 imply that for $n \geqslant 8$ and $n=3$ an arbitrary $(n-2)$ eigenfunction of $S_{n}$ with the minimum cardinality of the support is the difference of the characteristic functions of two completely regular codes of covering radius 2 . It is very interesting that there is an analogue of this fact for the Hamming graph $H(n, q)$ (see [18], Theorem 3). Namely, an arbitrary eigenfunction of $H(n, q)$ corresponding to the second largest eigenvalue with the minimum cardinality of the support is the difference of the characteristic functions of two completely regular codes of covering radius 1 .

\section{Concluding remarks}

The initial problem of finding $(n-2)$-eigenfunctions of $S_{n}$ with the minimum size of the support is formulated for arbitrary real-valued functions from corresponding eigenspace. Surprisingly, Theorem 20 implies that such functions take only three distinct values. It is interesting that the same fact holds for the Doob graph, for the Hamming graph and for the Johnson graph (see $[3,20,22])$. But, in general case it is not true. For example, in the Petersen and Desargues graphs there are $(-2)$-eigenfunctions with the minimum sizes of the supports that take five distinct values (see [16], Figure 3 and 9).

We note that the restrictions for $n$ in Theorem $20(n \geqslant 8$ or $n=3)$ arise from the proofs of Lemmas 12, 15 and 16.

\section{Acknowledgements}

The authors are grateful to Sergey Goryainov for fruitful discussions. The authors are grateful to the referees for useful remarks. 


\section{References}

[1] S. B. Akers, B. Krishnamurthy. A group-theoretic model for symmetric interconnection networks. IEEE Trans. Comput., 38(4):555-566, 1989.

[2] S. V. Avgustinovich, E. N. Khomyakova, E. V. Konstantinova. Multiplicities of eigenvalues of the Star graph. Siberian Electronic Mathematical Reports, 13:12581270, 2016.

[3] E. A. Bespalov. On the minimum supports of some eigenfunctions in the Doob graphs. Siberian Electronic Mathematical Reports, 15:258-266, 2018.

[4] J. Borges, J. Rifà, V. A. Zinoviev. On completely regular codes. Problems of Information Transmission, 55(1):1-45, 2019.

[5] G. Chapuy, V. Feray. A note on a Cayley graph of Sym ${ }_{n}$ arXiv:1202.4976v2, 1-5, 2012.

[6] C. D. Godsil. Algebraic Combinatorics. Chapman and Hall Mathematics Series, Chapman Hall, New York, 1993.

[7] S. Goryainov, V. Kabanov, L. Shalaginov, A. Valyuzhenich. On eigenfunctions and maximal cliques of Paley graphs of square order. Finite Fields and Their Applications, 52:361-369, 2018.

[8] S. Goryainov, V. Kabanov, E. Konstantinova, L. Shalaginov, A. Valyuzhenich. PIeigenfunctions of the Star graphs. Linear Algebra and its Applications, 586:7-27, 2020.

[9] J. S. Jwo, S. Lakshmivarahan, S. K. Dhall. Embedding of cycles and grids in star graphs. J. Circuits, Syst., Comput., 1(1):43-74, 1991.

[10] E. N. Khomyakova, E. V. Konstantinova. Note on exact values of multiplicities of eigenvalues of the Star graph. Siberian Electronic Mathematical Reports, 12:92-100, 2015.

[11] E. N. Khomyakova. On the eigenvalues multiplicity function of the Star graph. Siberian Electronic Mathematical Reports, 15:1416-1425, 2018.

[12] R. Krakovski, B. Mohar. Spectrum of Cayley graphs on the symmetric group generated by transpositions. Linear Algebra and its Applications, 437(3):1033-1039, 2012.

[13] D. S. Krotov. Trades in the combinatorial configurations. XII International Seminar Discrete Mathematics and its Applications, Moscow, 20-25 June 2016, 84-96 (in Russian).

[14] D. S. Krotov, I. Yu. Mogilnykh, V. N. Potapov. To the theory of q-ary Steiner and other-type trades. Discrete Mathematics, 339(3):1150-1157, 2016.

[15] V. N. Potapov. On perfect 2-colorings of the q-ary n-cube. Discrete Mathematics, 312(6):1269-1272, 2012.

[16] E. V. Sotnikova. Eigenfunctions supports of minimum cardinality in cubical distanceregular graphs. Siberian Electronic Mathematical Reports, 15:223-245, 2018. 
[17] E. V. Sotnikova. Minimum supports of eigenfunctions in bilinear forms graphs. Siberian Electronic Mathematical Reports, 16:501-515, 2019.

[18] A. Valyuzhenich. Minimum supports of eigenfunctions of Hamming graphs. Discrete Mathematics, 340(5):1064-1068, 2017.

[19] A. Valyuzhenich. Eigenfunctions and minimum 1-perfect bitrades in the Hamming graph. arXiv:2003.01571, March 2020.

[20] A. Valyuzhenich, K. Vorob'ev. Minimum supports of functions on the Hamming graphs with spectral constraints. Discrete Mathematics, 342(5):1351-1360, 2019.

[21] K. V. Vorobev, D. S. Krotov. Bounds for the size of a minimal 1-perfect bitrade in a Hamming graph. Journal of Applied and Industrial Mathematics, 9(1):141-146, 2015.

[22] K. Vorob'ev, I. Mogilnykh, A. Valyuzhenich. Minimum supports of eigenfunctions of Johnson graphs. Discrete Mathematics, 341(8):2151-2158, 2018. 
Corrigendum - Added November 19, 2020 error.

The mention of SB RAS N I.5.1, project No.0314-2019-0016 on page 1 was added in 